\title{
Competencias Matemáticas a través de la implementación de actividades provocadoras de modelos
}

\section{Mathematical Competences through the Implementation of Model Eliciting Activities}

\author{
Verónica Vargas Alejo ${ }^{1}$ \\ César Cristóbal Escalante ${ }^{2}$ \\ Guadalupe Carmona ${ }^{3}$
}

\begin{abstract}
Resumen. Reflexión sobre las competencias matemáticas mostradas por estudiantes de bachillerato cuando realizaron una Actividad Provocadora de Modelos. La pregunta de investigación que guía la discusión es ¿qué competencias exhiben los estudiantes en la realización de actividades cercanas a la vida real? La perspectiva de Modelos y Modelación y el concepto de competencias de Kilpatrick integran el marco teórico. Mediante el análisis de datos se identificaron las competencias de comprensión conceptual, fluidez procedimental, competencia estratégica, razonamiento adaptativo y disposición productiva.
\end{abstract}

Palabras Clave: Actividad Provocadora de Modelos; Modelos y Modelación; Proporcionalidad; Competencias matemáticas.

Abstract: We reflect on the mathematical competences that high school students exhibited when they carried out a Model Eliciting Activity. The research question that guides the discussion is what competencies do students exhibit in solving

Fecha de recepción: 6 de marzo de 2017. Fecha de aceptación: 30 de octubre de 2017

1 Matemática Educativa/CUCEI, Universidad de Guadalajara, veronica.vargas@academicos.udg.mx

2 División de Ciencias e Ingeniería, Universidad de Quintana Roo, cescrist@uqroo.edu.mx

${ }^{3}$ Department of Interdisciplinary learning and teaching, College of Education and Human Development, Universidad de Texas en San Antonio, Guadalupe.Carmona@utsa.edu 
activities close to the real life? The Models and Modeling perspective and the Kilpatrick concept of competencies are part of our theoretical framework. Through the data analysis, we identified the competences of conceptual understanding, fluency, strategical competence, adaptive reasoning and productive disposition.

Keywords: Model Eliciting Activity, Models and Modeling perspective, Proportionality, Mathematical competences.

\section{INTRODUCCIÓN}

Hoy en día la sociedad demanda la formación de individuos con habilidades que les permita desempeñarse no sólo en la escuela, sino en su entorno cotidiano como profesionistas y ciudadanos responsables (National Council of Teachers of Mathematics, 2003). Los individuos deben participar con soluciones o propuestas para diversas problemáticas como la contaminación, la falta de producción de alimentos y la carencia de medios de transporte.

Los planes y programas educativos, derivados de la Reforma Integral de la Educación Media Superior en México, reconocen esta demanda y señalan la importancia de preparar a los alumnos para desenvolverse en diversos contextos a lo largo de la vida, comprender el mundo e influir en él, aprender de forma autónoma, desarrollar relaciones armónicas con quienes les rodean y participar con eficacia en su vida social, profesional y política (Secretaría de Educación Pública, 2016). Ello implica preguntarse ¿qué papel juega la matemática en la adquisición de competencias? ¿Qué significa desarrollar competencias matemáticas? ¿Cómo desarrollarlas?

Aprender matemáticas es un proceso en donde los individuos deben desarrollar comprensión de conceptos, dominio de procedimientos y habilidades matemáticas en la medida en que abordan situaciones que les demandan poner en juego sus conocimientos, habilidades, competencias e integrar experiencias (Lesh y Doerr, 2003a). Sin embargo, muchos de los problemas que se proponen en los cursos de matemáticas conducen a una única solución numérica, la cual se encuentra mediante la aplicación de algún algoritmo. De acuerdo con Santos (2007) no es frecuente que dentro de las actividades realizadas en el aula se promueva la discusión de estrategias entre los estudiantes para resolver 
problemas, ni la argumentación de la validez de soluciones derivadas de los procedimientos. ¿Cómo influyen estos aspectos en el desarrollo de competencias matemáticas por los estudiantes? ¿Qué otro tipo de actividades deberían llevar a cabo los alumnos?

En este artículo se reflexiona sobre la pregunta de investigación ¿qué competencias matemáticas exhiben los estudiantes de reciente ingreso al nivel medio superior cuando enfrentan situaciones cercanas a la vida real? El objetivo es conocer el tipo de competencias matemáticas que muestran los alumnos de bachillerato cuando llevan a cabo una Actividad Provocadora de Modelos (APM), en particular la llamada Gigante Bondadoso, también denominada Pie Grande.

Lesh y Doerr (2003a) describen las actividades de matematización de estudiantes de nivel primaria cuando realizan la APM Pie Grande, pero no mencionan investigaciones relacionadas con lo que ocurre cuando alumnos de otros niveles educativos la realizan. Tampoco hemos hallado literatura de investigación que muestre un análisis de las actividades de matematización exhibidas por estudiantes mexicanos de nivel primaria, ni en otro nivel educativo. Lo anterior conduce a preguntarnos cuáles son los procedimientos que emprenden los alumnos mexicanos y qué competencias exhiben, principalmente los estudiantes de reciente ingreso al bachillerato. El concepto de competencias matemáticas fue tomado de Kilpatrick (2002).

\section{MARCO CONCEPTUAL}

La literatura de investigación revisada fue la relacionada con la perspectiva de Modelos y Modelación (Lesh, 2010; Lesh y Doerr, 2003a; Lesh y Doerr, 2003b) y la de competencias matemáticas de Kilpatrick (2002, 2009, 2014). Ambas coinciden en proponer una formación de individuos con un enfoque distinto al tradicional (memorístico, algorítmico y caracterizado por propiciar un dominio de conocimiento disciplinar fragmentado o aislado entre sí) que implique el aprendizaje de conceptos matemáticos, la interrelación entre ellos y habilidades para utilizarlos en la solución de problemas. Estas perspectivas señalan que los estudiantes deben aprender a formular y resolver problemas, enfrentar situaciones y realizar actividades. En particular, la perspectiva de Modelos y Modelación propone llevar a cabo en el aula situaciones como las APM. 
Desarrollar competencias matemáticas, de acuerdo con De Corte (2007), implica adquirir conocimientos básicos accesibles, auto-organizados y flexibles, métodos heurísticos, meta-conocimiento, creencias positivas relacionadas con la disciplina y habilidades auto-regulatorias; implica que el estudiante sea capaz de transferir habilidades y conocimiento a nuevas tareas y contextos de aprendizaje.

Ser competente en matemáticas, de acuerdo con Kilpatrick (2009), significa que los estudiantes deben

- aprender con comprensión conceptos matemáticos, operaciones y relaciones matemáticas (comprensión conceptual),

- adquirir habilidad para llevar a cabo procedimientos de una manera flexible, eficaz, eficiente y apropiada (fluidez procedimental),

- aprender a formular, representar y resolver problemas matemáticos (competencia estratégica),

- desarrollar capacidad para explicar y justificar, de manera lógica y reflexiva, su propia forma de pensar (razonamiento adaptativo), y

- adquirir una concepción de la matemática como disciplina sensible, útil y valiosa, confianza en las propias capacidades, y el reconocimiento de que con diligencia y esfuerzo se logrará encontrar alguna solución (disposición productiva).

Estas cinco competencias no son independientes entre sí, sino que están relacionadas de manera compleja. El desarrollo de competencias requiere la creación de un proceso de aprendizaje activo/constructivo, auto regulado, situado y colaborativo, donde se considere que es el estudiante quien construye significados y conocimientos matemáticos (De Corte, 2007). Se necesita poner atención en el papel del profesor en el aula, el tipo de tareas a proponer y el ambiente de aprendizaje a generar.

La perspectiva de Modelos y Modelación (PMM) plantea la necesidad del uso de situaciones abiertas en las clases de matemáticas porque permiten a los estudiantes el desarrollo de conocimiento y habilidades para construir modelos e interpretaciones. Las Actividades Provocadoras de Modelos (APM o MEA por sus siglas en inglés: Models Eliciting Activities) son algunas de las situaciones que se proponen como generadoras de modelos y por lo tanto de conocimiento. Al abordar una APM los individuos crean una interpretación acerca de la situación, un modelo de ella, ligado a sus conocimientos previos. 


\section{De acuerdo con Lesh y Doerr (2003a):}

Los modelos son sistemas conceptuales (que consisten de elementos, relaciones y reglas que gobiernan las interacciones) expresados mediante el uso de sistemas de notación externa, y utilizados para construir, describir, o explicar los comportamientos de otros sistemas - de tal forma que el otro sistema pueda ser manipulado o predicho de manera inteligente. (p. 10)

Aprender matemáticas no es un proceso lineal, puede describirse como un proceso de desarrollo de sistemas conceptuales o modelos que se modifican de manera constante durante la interacción de un individuo o estudiante con una situación problemática o con un problema (Lesh y Yoon, 2004).

Los modelos se pueden compartir, manipular, modificar y reutilizar para describir, interpretar, construir, manipular, predecir o controlar sistemas matemáticamente significativos (Lesh, 2010). Son personales, porque reflejan la experiencia del estudiante o individuo al enfrentar situaciones y, por lo tanto, son posiblemente únicos.

Los sistemas conceptuales que construye una persona sobre una situación evolucionan de ser simples, burdos y poco articulados a ser modelos estructurados y complejos. De igual manera las representaciones para expresarlos y comunicarlos pueden ser diagramas, esquemas, descripciones (matemáticas o sólo con palabras), ecuaciones, entre otras; pueden ser simples o complejas y no siguen un orden de uso, aunque los dibujos tienden a ser los más utilizados (Lesh y Doerr, 2003a).

El proceso de aprendizaje de las matemáticas implica la construcción de ciclos progresivos (como espirales crecientes) de entendimiento, modificación, extensión y refinamiento de sistemas conceptuales. En estos ciclos los sujetos relacionan, con diversos niveles de profundidad, los datos, metas y posibles rutas de solución al enfrentar una situación problemática (Lesh, 2010). Los modelos construidos por un individuo en los primeros ciclos de entendimiento son burdos, en general, pero debido a que se refinan de manera paulatina, son parecidos a un organismo vivo, a un sistema dinámico, el cual se adapta y autorregula de manera continua (Lesh y Yoon, 2004). El desarrollo de conocimiento sobre conceptos y habilidades sigue un proceso evolutivo que va desde los sistemas conceptuales burdos, poco estructurados a sistemas conceptuales más robustos, organizados y formalizados, acordes con las experiencias de la persona. 
El proceso de desarrollo de conocimiento es un proceso social, en el cual se construyen y modifican modelos mediante las fases de diferenciación, integración y refinamiento de los diferentes sistemas que se van construyendo (Lesh y Doerr, 2003b). La comprensión de los conceptos se incrementa y robustece en la medida en que el individuo comunica y comparte sus modelos con otras personas y considera a los conceptos en diversas dimensiones: concreto - abstracto, particular - general, en contexto - sin contexto, intuitivo- analítico- axiomático, fragmentado- integrado, etcétera. El producto del aprendizaje no es el modelo sino el proceso de creación del modelo.

Pensar matemáticamente va más allá de hacer cálculos, "con frecuencia implica describir situaciones matemáticamente" (Lesh y Doerr, 2003a; p. 15). Significa analizar información cualitativa, cuantificarla en caso de ser necesario, atribuir dimensiones al espacio y ubicar eventos en marcos de referencia. Esto es difícil de lograr si en el salón de clases sólo se resuelven ejercicios. El desarrollo de comprensión de conceptos no se efectúa aislando cada concepto de otros, requiere un proceso mediante el cual se interrelacionen conceptos y se desarrollen habilidades para utilizarlos en el análisis y la interpretación de diversas situaciones (English, Lesh y Fennewald, 2008). En este sentido, el conocimiento desarrollado por una persona se refleja, no sólo en la descripción de los conceptos, los procedimientos y los cálculos utilizados, sino en la explicación de las situaciones enfrentadas.

La perspectiva de Modelos y Modelación recomienda propiciar la resolución de situaciones cercanas a la vida real en el aula que demanden la construcción de modelos y no sólo respuestas cortas a preguntas específicas. Las APM son situaciones cercanas al mundo real que requieren de la construcción de modelos, cuyo propósito es apoyar la toma de decisiones de un cliente. La solución no es una cantidad numérica, es un modelo en el cual el profesor puede identificar lo que los alumnos piensan acerca de la situación dada. El proceso de construcción del modelo ayuda a los estudiantes a desarrollar conocimiento, de ciencia, ingeniería y otras disciplinas. Los modelos y el proceso de construcción permiten, además, identificar los conceptos, procedimientos y habilidades (Lesh y Doer, 2003b) de los alumnos.

La comunicación de los modelos permite a los estudiantes modificar y extender sus ciclos de entendimiento y mejorar su aprendizaje de los conceptos. Por lo tanto, es importante propiciar que los alumnos comuniquen sus interpretaciones en diferentes formas y contextos (por escrito, en forma verbal) a sus compañeros y al instructor (Lesh, Cramer, Doerr, Post y Zawojewsky, 2003). 


\section{METODOLOGÍA}

La metodología utilizada para el análisis de los datos fue de tipo cualitativa, la investigación se enfocó en conocer y comprender el comportamiento natural de los estudiantes de reciente ingreso al bachillerato, cuando realizan una actividad cercana a la vida real; es decir, se buscó observar e interpretar las respuestas, discursos y discusiones de los alumnos y su relación con las competencias matemáticas.

La recolección de datos se hizo mediante las producciones escritas por los estudiantes y las observaciones del profesor registradas en una bitácora. Se analizaron los modelos de solución construidos y explicados por los alumnos.

\subsection{LA POBLACIÓN DE ESTUDIO}

En este estudio participó un grupo de 40 estudiantes mexicanos, cuyas edades se encontraban en el intervalo de 15 a 16 años. Quienes tomaban un curso propedéutico para el ingreso al bachillerato, por ello sus conocimientos matemáticos correspondían al nivel de un egresado de secundaria. Al momento de resolver el problema GB contaban con la experiencia de haber abordado, en ambientes colaborativos, aproximadamente 12 problemas matemáticos típicos de libros de texto, durante cinco sesiones de dos horas cada una. El conocimiento matemático requerido para resolverlos fue razones, proporciones, ecuaciones lineales, funciones lineales, sistemas de ecuaciones lineales y algunas medidas de tendencia central.

\subsection{El PROBLEMA DEL GIGANTE BONDADOSO}

El problema cuyos modelos se discuten en este artículo es el Gigante Bondadoso (GB), adecuación realizada por Carmona (2015) de la actividad provocadora de modelos Pie Grande (Lesh y Doerr, 2003a). Los conceptos matemáticos centrales son razones, proporciones y productos.

El problema formó parte de las actividades de un proyecto de investigación, diseñadas para desarrollar conocimientos (razones, variación, proporción, funciones y ecuaciones) y habilidades matemáticas. 
De acuerdo con Lesh (1997) las APM como Pie Grande (problema GB) involucran a los estudiantes en actividades de matematización, como cuantificar y asignar dimensiones.

Es similar a un problema de paleontología que involucra estimar qué tan rápido una especie particular de dinosaurio podría correr sólo a partir de un análisis de fotografías de huellas descubiertas en un sitio arqueológico de Utah. La idea matemática central, que los estudiantes deben utilizar en el problema de Pie Grande, involucra razonamiento proporcional -quizás basado en las relaciones lineales tales como $Y=S^{*} X$ (Lesh y Doerr, 2003a, p. 5).

La Actividad Provocadora de Modelos el Gigante Bondadoso (Figura 1) se presentó a los alumnos en cinco hojas tamaño carta. Las dos primeras contenían un artículo periodístico con preguntas de comprensión lectora, la tercera incluyó el problema y las dos últimas la huella del gigante. El objetivo del artículo fue que los estudiantes se familiarizaran con el contexto del problema.

\section{VOZ VIVIENTE}

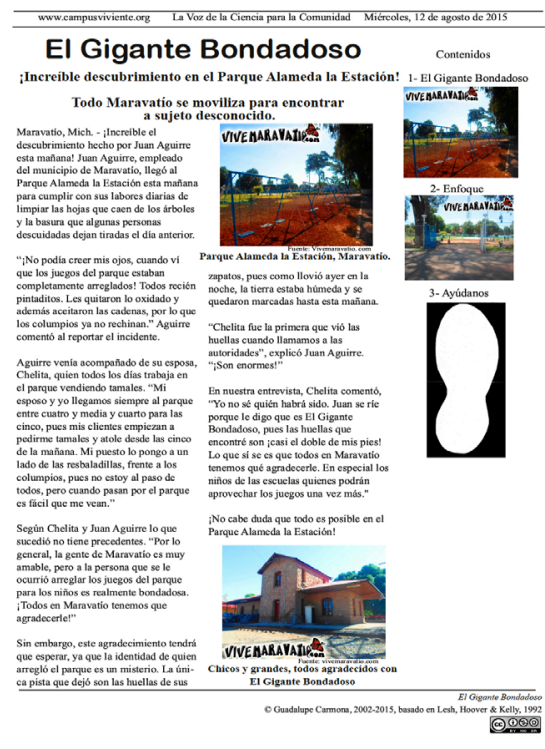

ENFOQUE

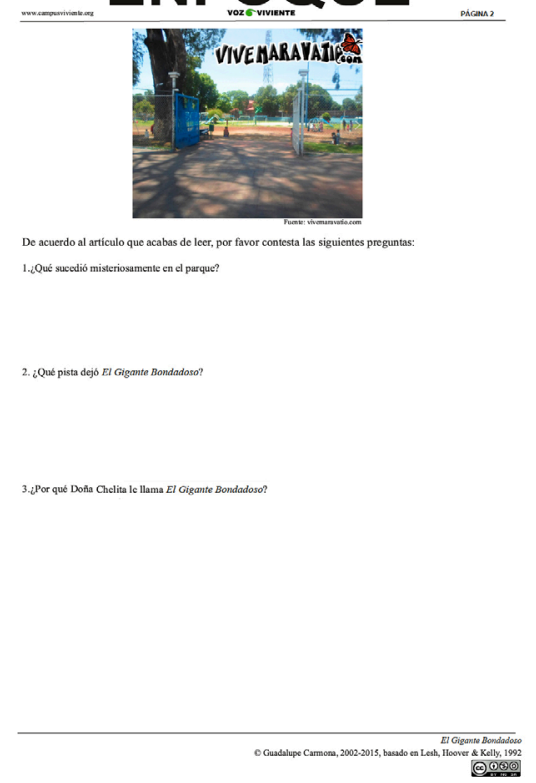




\section{AYÚDANOS}
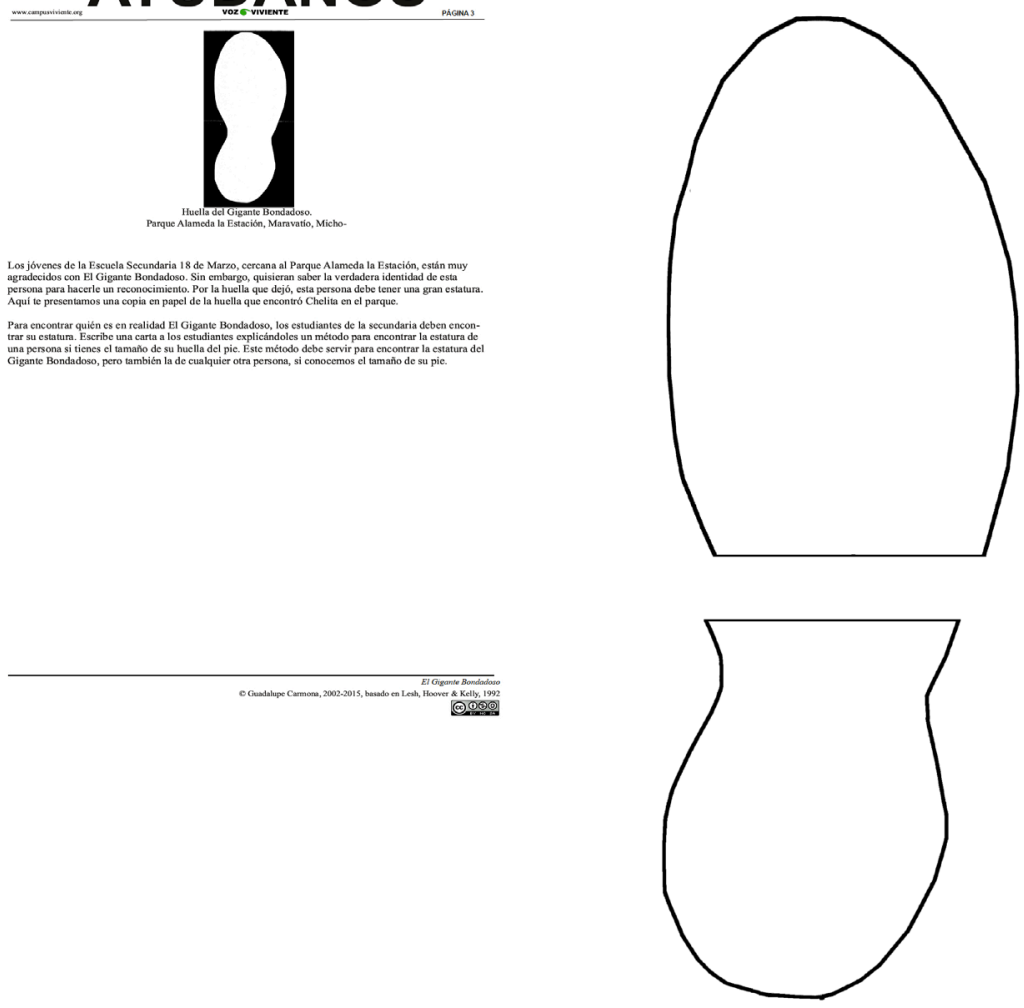

Figura 1. Problema el Gigante Bondadoso.

Problema: El Gigante Bondadoso. Los jóvenes de la Escuela Secundaria 18 de Marzo, cercana al Parque Alameda la Estación, están muy agradecidos con el Gigante Bondadoso. Sin embargo, quisieran saber la verdadera identidad de esta persona para hacerle un reconocimiento. Quien por la huella que dejó, debe ser de gran estatura. Aquí te presentamos una copia en papel de la huella que encontró Chelita en el parque. Para saber quién es en realidad el Gigante Bondadoso, los estudiantes de la secundaria deben calcular su estatura. Escribe una carta a los estudiantes explicándoles un método para determinar la estatura de una persona si tienes la longitud de su huella del pie. Este método debe servir para encontrar la estatura del Gigante Bondadoso, pero también la de cualquier otra persona, si conocemos la longitud de su pie. 


\subsection{PROCESO DE RESOLUCIÓN DEL PROBLEMA}

El proceso realizado en el aula para resolver el problema se desarrolló en cuatro fases.

a) Entrega del artículo periodístico a los siete equipos y aclaración de dudas.

b) Resolución del problema en equipos, en ambiente colaborativo. Se les indicó que podían utilizar los materiales (cintas métricas, papel y cinta adherible) que estaban en una mesa al frente del salón.

c) Escritura de la carta y preparación de exposición. Cada equipo escribió su solución en una carta dirigida al cliente.

d) Presentación de la carta en el grupo, evaluación y discusión de los modelos. El grupo escuchó y discutió las soluciones numéricas y los modelos planteados de acuerdo con las necesidades del cliente.

El docente participó como observador durante todo el proceso y como facilitador de la discusión grupal. Durante la resolución del problema aclaró dudas mediante el planteamiento de preguntas como las siguientes: no entendí tu pregunta, ¿podrías plantearla de nuevo de manera diferente?, ¿qué se pide en el problema?, ¿qué información se proporciona? El profesor evitó hacer comentarios que pudieran orientar a los equipos hacia una solución específica. Las preguntas tenían como objetivo identificar las competencias de los estudiantes. La manera como se llevó a cabo la actividad fue diferente a la propuesta por Lesh y Doerr (2003a) porque hubo interacción del docente con los estudiantes.

\subsection{CRITERIOS DE EVALUACIÓN}

Para el análisis de los datos se utilizó la clasificación de competencias de Kilpatrick (2009): comprensión conceptual, fluidez procedimental, competencia estratégica, razonamiento adaptativo y disposición productiva. Estas competencias se detectaron durante la resolución del problema y la comunicación de los modelos en la fase de discusión grupal. La resolución del problema se describe en los ciclos de entendimiento cualitativo y cuantitativo (Vargas-Alejo, Reyes-Rodríguez y Cristóbal-Escalante, 2016).

Se entenderá por ciclo de entendimiento cualitativo, aquel en el cual el estudiante empieza a tomar sentido del problema GB y las variables 
involucradas. El alumno puede utilizar metáforas basadas en la experiencia, observar la existencia de variables y cierta relación entre ellas, sin necesariamente establecer algún tipo de relación en forma numérica, tabular, gráfica o algebraica. La comparación entre variables puede ser cualitativa, por ejemplo, un estudiante puede interpretar que realizar la actividad GB implica "analizar la longitud y el ancho del pie". A la estatura la puede considerar, sin mencionarlo explícitamente, como una variable que puede tomar valores "pequeños" o "grandes".

El ciclo de entendimiento se vuelve cuantitativo cuando el estudiante es capaz de establecer qué significa "grande", "pequeño"; es decir, cuando el estudiante puede responder preguntas como "¿qué tan grande o pequeño es?" y las comparaciones se vuelven numéricas. En ese momento los datos pueden organizarse en representaciones tabulares y gráficas.

Al modelar, los estudiantes transitan a través de múltiples ciclos de descripciones, interpretaciones, conjeturas y explicaciones que se refinan iterativamente. Alcanzar la comprensión o entendimiento conceptual no se logra a través de un proceso lineal. Los ciclos de entendimiento pueden coexistir a la vez, en ellos se observan fases de diferenciación, integración y refinamiento, que muestran los avances de los estudiantes y también retrocesos en la comprensión conceptual.

\section{RESULTADOS Y SU DISCUSIÓN}

De acuerdo con Lesh y Doerr (2003a) el problema ha provocado en estudiantes de educación primaria la generación de diversas representaciones, que van desde comparaciones aditivas hasta comparaciones de tipo multiplicativas y razonamientos proporcionales basados en relaciones lineales (de modelos burdos a modelos complejos). Por lo tanto se esperaba que los estudiantes participantes en este estudio, dada su mayor experiencia matemática, exhibieran modelos complejos que incluyeran conocimientos algebraicos.

Sin embargo, no se tenía certeza respecto a la forma cómo los estudiantes de bachillerato, a diferencia de los de primaria, formularían, representarían y resolverían la situación y qué conceptos matemáticos, operaciones y relaciones utilizarían. 


\subsection{COMPETENCIAS EXHIBIDAS}

El primer ciclo de entendimiento de los equipos de estudiantes fue cualitativo. Consistió en utilizar la huella impresa y compararla directamente con su pie. Algunas expresiones fueron "es muy grande", "debe ser una persona muy alta".

Enseguida, algunos equipos empezaron a observar, medir y comparar la longitud de los pies con la estatura respectiva de alguno o varios de los integrantes de su equipo. Es decir, su ciclo de entendimiento se transformó en cuantitativo.

Este nuevo ciclo de entendimiento se caracterizó por la búsqueda de una solución numérica. Los estudiantes transformaron la pregunta del problema "¿cómo desarrollar un método para encontrar la estatura de una persona cuando la única información que se tiene es la copia de una huella?" en "¿cuál es la altura de la persona cuya huella se presenta en esta actividad?"

Los equipos emprendieron procedimientos que requerían varios conceptos matemáticos, operaciones y relaciones (Tabla 1 ) para resolver el problema. Uno de los siete equipos (A) utilizó proporciones. Tres de los siete equipos (B, C, D) utilizaron proporciones y conocimiento de estadística (así lo explicaron los equipos y se refirieron al hecho de tomar a los miembros de su equipo como una muestra de la población, medir la longitud de sus pies, las estaturas correspondientes y calcular promedios). Los equipos E, F y G dijeron que el problema no tenía solución. La Tabla 1 resume las competencias conceptuales exhibidas antes de la discusión grupal.

Tabla 1. Competencias conceptuales de los equipos de estudiantes, evidenciadas al inicio del proceso de resolución del Problema GB.

\begin{tabular}{|c|l|}
\hline Equipos & \multicolumn{1}{|c|}{ Competencia conceptual } \\
\hline E, F, G & $\begin{array}{l}\text { No lograron utilizar, ni integrar conceptos matemáti- } \\
\text { cos para resolver el problema. Consideraron que el } \\
\text { problema no tenía solución. }\end{array}$ \\
\hline A & Uso de proporciones. \\
\hline B, C, D & Uso de proporciones y conocimiento de Estadística. \\
\hline
\end{tabular}

Enseguida se muestran las cartas escritas por los equipos de estudiantes y presentadas en la discusión grupal. Se observa cómo relacionaron, con diversos 
niveles de profundidad, los datos, metas y posibles rutas de solución cuando se enfrentaron a la situación problemática. El orden en el que se muestran las cartas corresponde al orden de exposición de las mismas durante la discusión grupal.

Los equipos E y G no presentaron sus cartas al grupo porque no las tenían escritas antes de la discusión grupal.

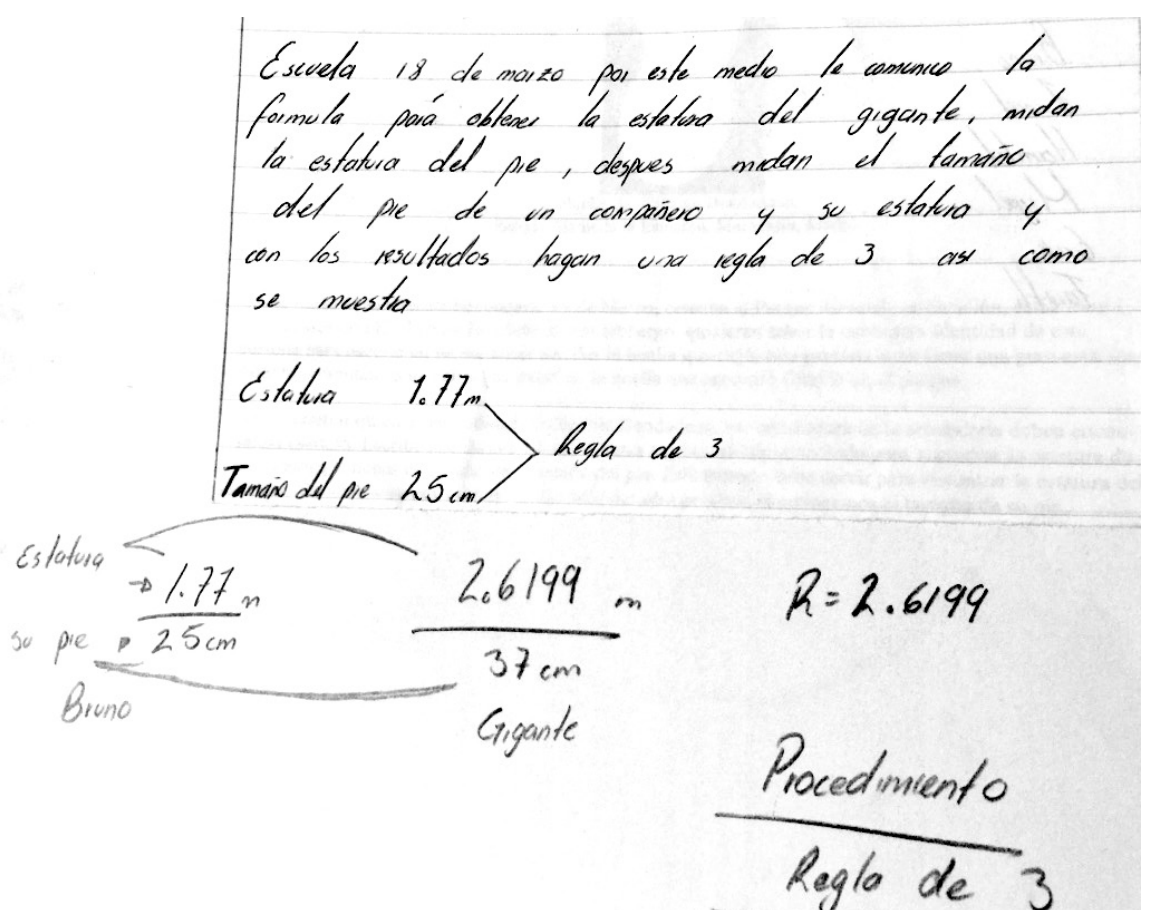

Figura 2. Carta del equipo A.

La carta del equipo A contiene algunas frases que permiten entender su modelo. Escribieron: "por este medio le comunico la fórmula para obtener la estatura del gigante. Midan la estatura [la longitud] del pie, después midan el tamaño del pie de un compañero y su estatura y con los resultados hagan una regla de 3 así como se muestra". En su carta describieron un procedimiento (Figura 2), aunque en su exposición pusieron énfasis en el resultado.

Los alumnos identificaron una relación entre la longitud del pie y la estatura de una persona, y la describieron verbalmente de la siguiente manera: "una 
persona de baja estatura tiene un pie pequeño, mientras que una persona alta tiene un pie grande". Es decir, reconocieron un patrón. Mencionaron que su referente fue la estatura del compañero más alto de su grupo (de $1.77 \mathrm{~m}$ ) y el largo de su pie (de $25 \mathrm{~cm}$ ). Utilizaron proporciones y obtuvieron la estatura solicitada.

La longitud de la huella del Gigante $(37 \mathrm{~cm}$ ) les condujo a obtener una estatura de $2.61 \mathrm{~m}$. Este equipo justificó la elección del estudiante más alto como referente para la toma de medidas porque en el problema se menciona un gigante.

Los integrantes del equipo A evidenciaron competencia de fluidez procedimental (Tabla 2) en el uso de razones y proporciones, competencia estratégica, es decir, habilidades para formular, representar y resolver el problema. Mostraron razonamiento adaptativo o capacidad para explicar y justificar, de manera lógica y reflexiva, su propia forma de pensar y la competencia de disposición productiva; exhibieron confianza en sus conocimientos y capacidades, así como reconocimiento de que con diligencia y esfuerzo encontrarían la solución. Los estudiantes redactaron su carta de la siguiente manera (Figura 3).

iSaludos a los alumnos de la Sec. 18 de marzo!

Nos enteramos de que querían averiguar la identidad del "Gigante bondadoso" y para ello necesitaban saber su estatura.

Así que averiguamos una manera de sacar su estatura con la representación del pie que teníamos.

Nos dimos cuenta de que el pie del gigante bondadoso tenía una longitud de 36 $\mathrm{cm}$, así que nuestra idea para saber su altura, fue sacar una relación a la medida del pie y su altura, usando como referencia a 4 personas, sacando las medidas de su altura y su calzado, llegando a la conclusión de que por cada $\mathrm{cm}$ de la medida del pie, se le aumen (sic) $6.37 \mathrm{~cm}$ a la estatura de una persona.

Viendo que el pie del gigante tenía una medida de $36 \mathrm{~cm}$, según nuestros cálculos, su altura es de $229 \mathrm{~cm}$. (2.29m).

Esperamos les sirva de apoyo para encontrar al Gigante bondadoso.

Los integrantes del Equipo B utilizaron proporciones y conocimiento de estadística. Este modelo no aparece en la literatura de investigación presentada por Lesh y Doerr (2003a). 
iSaludas a los a lumnos de la Sec. 18 de marro! Nos enteramos de gue guerían averiguar la dentidad del "Tigante bondadoso, y para ello necestaban saber su estalera. con la replesentactor del pie que tentiamos

Nos dimos cuenta de gue el pre del Einante barda-

doso tenica una longitud de $36 \mathrm{~cm}$ ast que tuestra

dea para saber su altera, fue socar cra reloción ontr

la medida del ple y su alterer usando como

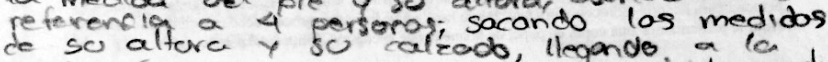
condusión de gue par roda con. de la medida del pie, se le aumen $6.37 \mathrm{~cm}$ a la estatera, de una persona.

Viendo que el pie del gigate tenía una medieda de $36 \mathrm{~cm}$, jegún nuestros calculos, so altura es de $229 \mathrm{~cm}$. (2.29 metros).

Esperamos les sirva de apoyo para encontrar al
Sigante bondadoso.

Figura 3. Carta del equipo B.

La carta de los estudiantes no contiene las operaciones matemáticas realizadas. Pero, en hojas anexas (Figura 4) las registraron y explicaron al grupo. Midieron la estatura de varios estudiantes y la longitud de su pie. Compararon estas medidas y las utilizaron para encontrar la estatura buscada del Gigante Bondadoso. Los datos 24, 25, 26.5 y 27 son las medidas correspondientes a la longitud de los pies de cada alumno utilizado como referente.



Figura 4. Operaciones complementarias a la carta del equipo B. 
Los alumnos interpretaron al signo igual como "corresponde a". Por ejemplo: 25=160 lo leyeron como: "la longitud del pie de $25 \mathrm{~cm}$ corresponde a una estatura de $160 \mathrm{~cm}$ ". Cambiaron estas relaciones en otras con valores unitarios (Vergnaud, 1991). Por ejemplo, la relación 27=172 (27 cm corresponden a 172 $\mathrm{cm}$ ) fue transformada en $1=6.37$ (uno corresponde a 6.37) y la relación 24=152 (24 corresponde a 152) en $1=6.33$ (uno corresponde a 6.33). Los alumnos calcularon el promedio de las cantidades: $6.37+6.33+6.40+6.40$. Su objetivo fue encontrar la relación entre las longitudes de los pies y las estaturas de las personas correspondientes, para posteriormente, usarla y encontrar la estatura del Gigante Bondadoso.

Los integrantes del equipo B explicaron lo siguiente durante la discusión grupal: "cada centímetro de la longitud del pie [de los estudiantes] equivale a $6.37 \mathrm{~cm}$ de la estatura [correspondiente]". Este dato lo utilizaron para calcular la estatura del Gigante Bondadoso: $36 \mathrm{~cm}$. En lugar de proporcionar un modelo al cliente, brindaron una solución numérica.

Evidenciaron competencia de comprensión conceptual más robusta que el equipo A, porque exhibieron conocimiento de estadística, aunque también proporcionaron una respuesta numérica y no un modelo como respuesta al problema. Su fluidez procedimental se manifestó en el uso adecuado de razones, proporciones y conocimiento de estadística. Los alumnos exhibieron competencia estratégica o habilidad para formular, representar y resolver el problema y razonamiento adaptativo o capacidad para explicar y justificar, de manera lógica y reflexiva, su propia forma de pensar. Evidenciaron confianza en sus conocimientos y capacidades. No descansaron hasta encontrar el mejor modelo, es decir, sabían que con diligencia y esfuerzo encontrarían la solución al problema. Esta descripción de las competencias de los integrantes del equipo B, aplica para los estudiantes de los equipos C y D, cuyo modelo se describe a continuación.

Los equipos C y D trabajaron juntos. Utilizaron proporciones y conocimiento de estadística. Elaboraron un modelo a partir de la sugerencia planteada por una de las estudiantes, quien explicó a sus compañeros cómo podrían resolver el problema. Su modelo lo llamaron "Regla de 3 y promedio".

Midieron la estatura de varios estudiantes y la longitud de su pie. Compararon estas medidas con la del pie del Gigante Bondadoso $(37 \mathrm{~cm})$ y mediante una regla de 3 obtuvieron la estatura buscada. Al final promediaron las posibles estaturas y encontraron 2.41 como resultado. La presentación de la carta al grupo permitió entender la solución (Figura 5). 


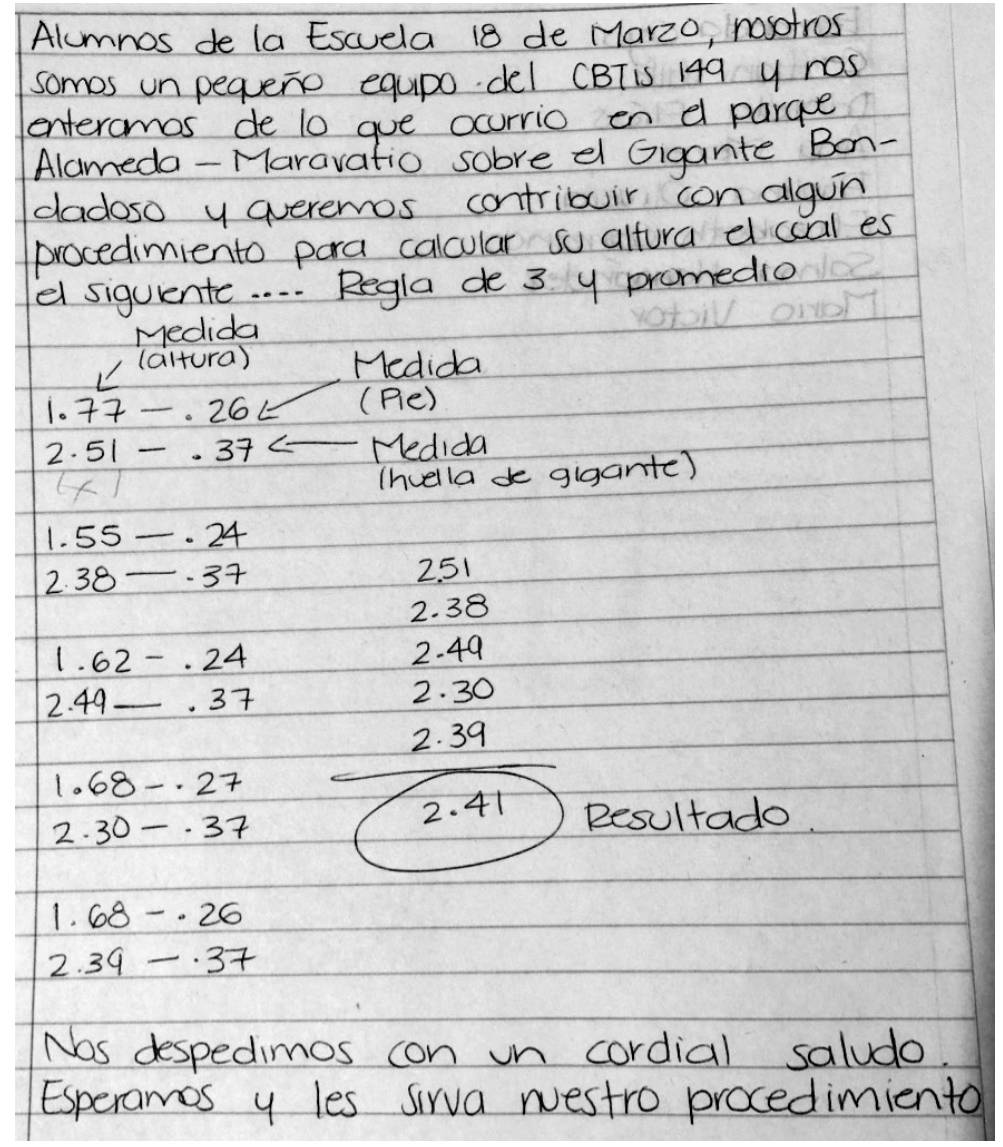

Figura 5. Carta de los equipos C y D.

El texto de la carta escrita por los equipos C y D es el siguiente: "Alumnos de la escuela 18 de marzo, nos enteramos de lo que ocurrió en el parque Alameda-Maravatío sobre el Gigante Bondadoso y queremos contribuir con algún procedimiento para calcular su altura el cual es el siguiente .... [...] Regla de 3 y promedio".

Los integrantes del equipo E utilizaron proporciones como se observa en su carta (Figura 6): "Compañeros de la Secundaria 18 [de marzo] en Maravatío, Mich les mandamos una solución que probablemente les ayude a saber la medida del gigante bondadoso: la medida obtenida fue 2.36 (utilizamos una regla de 3)". 


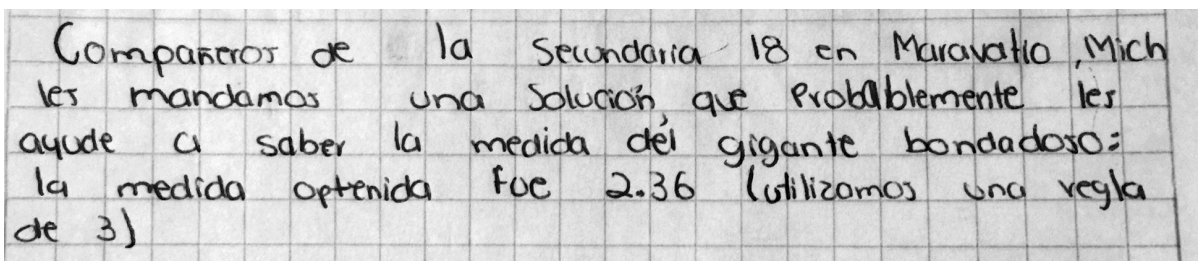

Figura 6. Carta del equipo E.

Previo a escribir esta carta, tuvieron muchas dificultades en cuanto a la comprensión del problema. Una estudiante de otro equipo les explicó que con una regla de 3 se podía obtener la solución. Esto influyó en la redacción de la carta, la cual no fue presentada durante la discusión grupal porque aún no estaba terminada.

Los estudiantes del equipo E evidenciaron competencia de comprensión conceptual limitada. Manifestaron falta de conocimientos matemáticos que pudieran utilizar para resolver el problema; su fluidez procedimental también fue pobre. Les faltaba competencia estratégica. Es decir, no estaban acostumbrados a formular, representar y resolver problemas matemáticos, ni a pensar, reflexionar, explicar y justificar de manera lógica; así lo manifestaron cuando solicitaron al docente una explicación sobre cómo resolver el problema, para evitar esforzarse (disposición productiva escasa). No tenían confianza en sus conocimientos y capacidades (razonamiento adaptativo limitado). Esta descripción aplica también para el equipo G (Figura 7).

El equipo G propuso una respuesta numérica como solución del problema. Tanto el contenido de la carta, como el modelo propuesto son difíciles de entender. Se presenta a continuación. Se eliminaron algunas faltas de ortografía.

Alumnos de secundaria del parque Alameda

Por medio de este presente hemos elaborado una posible respuesta al gigante bondadoso.

Por medio de un zapato con una elevación de un metro sacamos la posibilidad de que este ser mida 9.96.

Multiplicamos por año con la altura de un compañero y multiplicamos por año. 0 saquen el modelo del pie de cada comunidad de las personas. 


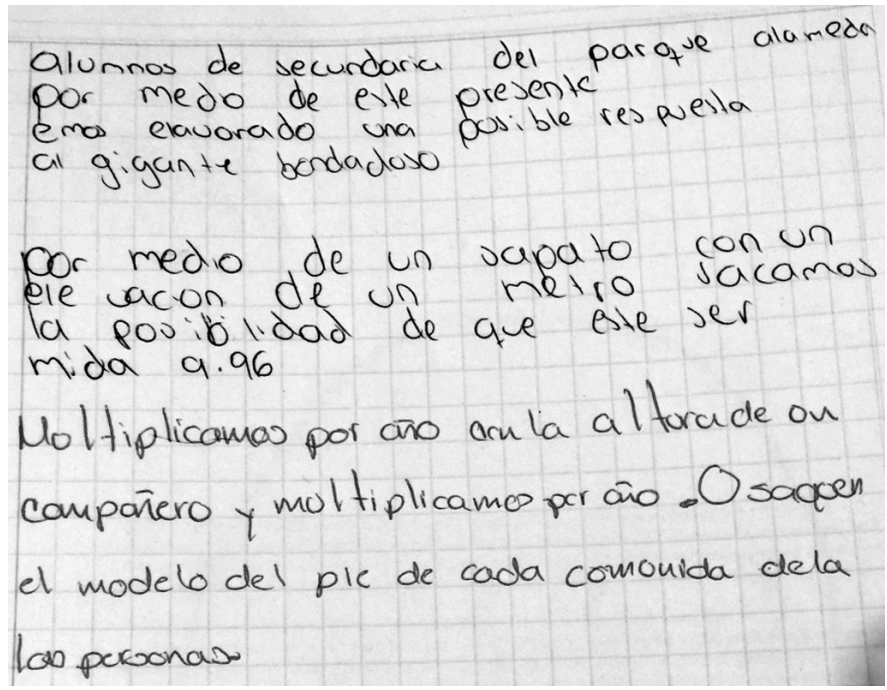

Figura 7. Carta del equipo G.

La frase "o saquen el modelo del pie de cada comunidad de las personas" se refiere a comparar la longitud del pie de cada individuo de la comunidad de Maravatío con su estatura. Es decir, los estudiantes adoptaron uno de los modelos explicados durante la sesión grupal. El equipo G no presentó la carta durante la discusión grupal porque aún no estaba terminada.

El equipo F no logró construir en equipo un modelo para resolver el problema. Dos de los integrantes del equipo F utilizaron operaciones de adición; es decir, calcularon la diferencia entre la longitud de su pie y la huella y trataron de relacionarla con la diferencia entre su estatura y la del Gigante Bondadoso. Sin embargo, no lograron estructurar, ni justificar sus ideas. Su forma de proceder parecía más aditiva que multiplicativa.

Otro de los estudiantes mencionó que era muy difícil determinar la medida del Gigante, porque la estatura de las personas y la longitud de los pies variaban mucho. Es decir, no existía una relación entre ambas medidas. Señaló que en su entorno había personas de baja estatura con pies muy grandes y viceversa. El contenido de la carta fue el siguiente (Figura 8): "Escuela 18 de marzo por este medio les comunicamos la fórmula de este proyecto para poder sacar el resultado del tema y la fórmula que utilizamos fue medir el pie de todos y el que fuera el más grande solo le sumamos la diferencia". Esta carta no fue presentada durante la discusión grupal porque aún no estaba terminada. 


\section{Escuela 18 aemareo par este medio les cumunicamos la farmula de este prayedo para poder scicar el resultado del tema y la farmula que utilizamas fue medir el pie de todos y el que foera el mas grande solo le sumamos la diferiencia}

\section{Estatura 2.28}

Figura 8. Carta del equipo F.

En el equipo F, a diferencia del E, hubo estudiantes que se negaron a utilizar conceptos matemáticos para resolver el problema. Ni las presentaciones del resto de los equipos los convencieron de que podían generar un modelo para enfrentar la situación (falta de razonamiento adaptativo).

En general, las exposiciones de los equipos A, B, C y D durante la discusión grupal fueron esenciales para la reflexión, modificación, extensión y refinamiento de sistemas conceptuales iniciales (Tabla 1); con ello también se puso a prueba la competencia estratégica de los estudiantes para explicar y justificar de manera lógica.

La comprensión conceptual de los equipos A, E, G mejoró durante la discusión grupal (Tabla 2). Por ejemplo, el equipo A consideró que pudo haber medido la longitud del pie y estatura de cada uno de sus integrantes y calculado el promedio. El equipo G incluyó en su carta uno de los modelos explicados.

Sin embargo, la comprensión conceptual de los estudiantes del equipo F no mejoró (Tabla 3). Los alumnos manifestaron inconformidad ante los modelos presentados durante la discusión grupal, aseguraron que era imposible determinar la estatura del Gigante Bondadoso. Su argumento permaneció invariante: "existen personas bajitas con pies muy grandes y personas altas con pies pequeños". La estimación no fue algo que pudiera proponerse como procedimiento matemático para resolver el problema. Argumentaron la falta de un algoritmo para resolverlo.

Los modelos propuestos por los equipos B y C para determinar la estatura del Gigante Bondadoso fueron considerados los mejores por todo el grupo (excepto el equipo F) porque respondían la pregunta del problema: ¿Cómo desarrollar un método para encontrar la altura de una persona cuando la única información que se tiene es la copia de una huella? Y no a la pregunta planteada por los equipos inicialmente: ¿Cuál es la altura de la persona cuya huella se presenta en el problema? 


\begin{tabular}{|c|c|c|c|c|}
\hline 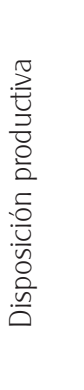 & \multicolumn{2}{|c|}{ 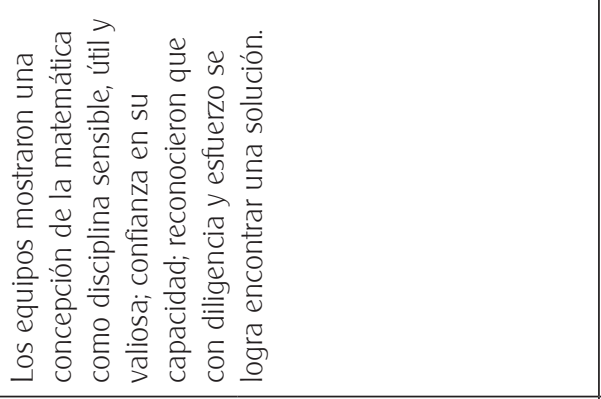 } & \multicolumn{2}{|l|}{ 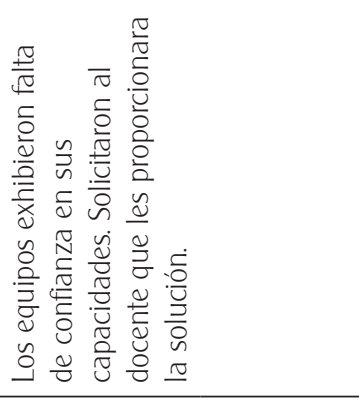 } \\
\hline 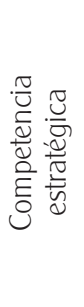 & \multicolumn{2}{|l|}{ 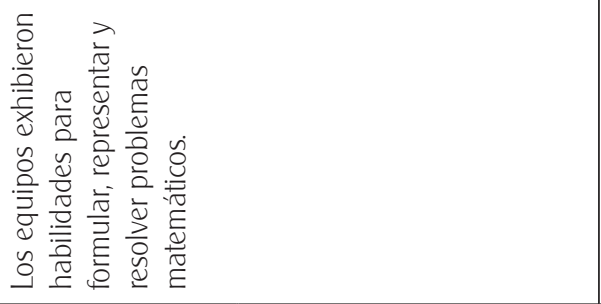 } & \multicolumn{2}{|c|}{ 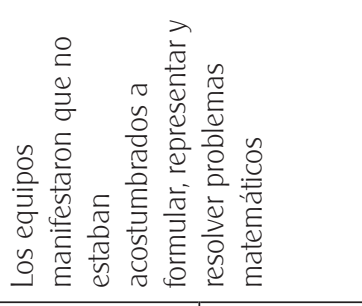 } \\
\hline 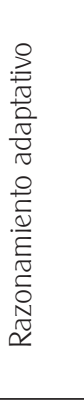 & 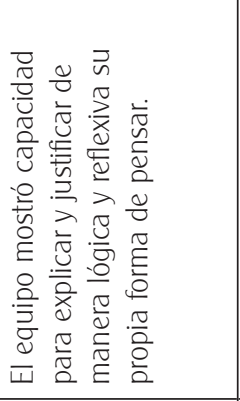 & 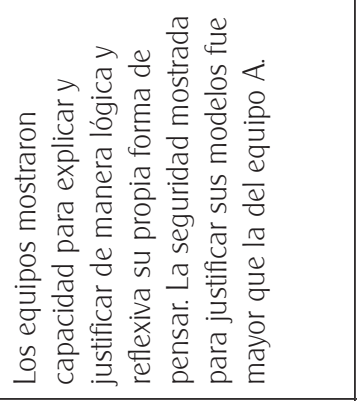 & 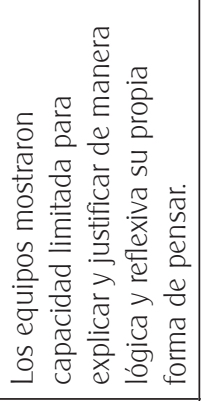 & 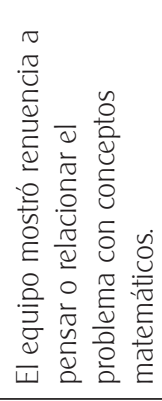 \\
\hline 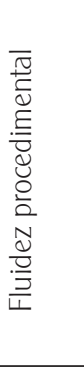 & 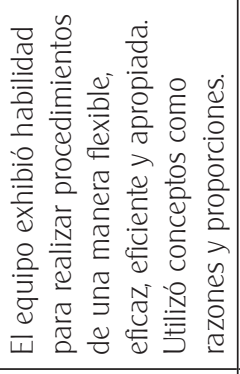 & 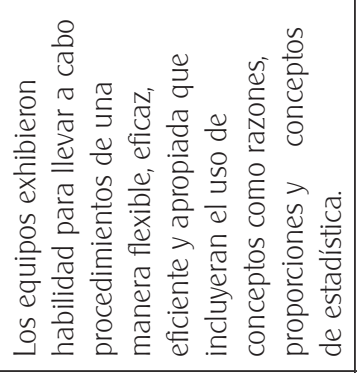 & 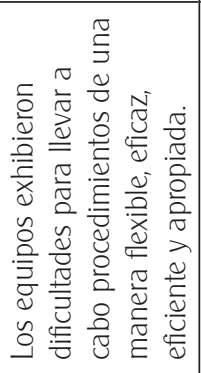 & 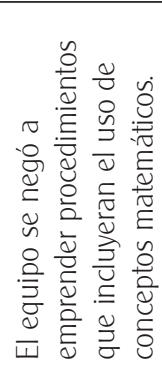 \\
\hline $\begin{array}{l}\tilde{O} \\
\stackrel{亏}{\bar{\Xi}} \\
\text { Ð }\end{array}$ & $\ll$ & $\underset{\infty}{\sim}$ & $\begin{array}{l}\circlearrowleft \\
\sqcup\end{array}$ & $\sqcup$ \\
\hline
\end{tabular}


Tabla 3. Competencia conceptual de los equipos de estudiantes al final del proceso de resolución del Problema GB.

\begin{tabular}{|c|l|}
\hline F & $\begin{array}{l}\text { No lograron utilizar ni integrar } \\
\text { conceptos matemáticos para resolver } \\
\text { el problema. }\end{array}$ \\
\hline A, B, C, & Uso de proporciones y conocimiento \\
D, E, G & de Estadística. \\
\hline
\end{tabular}

El significado de solución del problema cambió. Es decir, los equipos (con excepción del F) se enfocaron en proponer al cliente un modelo que generara un valor aproximado o un intervalo de valores posibles como estatura del Gigante Bondadoso, y no una solución numérica exacta.

\section{CONCLUSIONES}

¿Qué competencias matemáticas mostraron los estudiantes mexicanos recién inscritos en el nivel medio superior al llevar a cabo la Actividad Provocadora de Modelos GB? Los integrantes de los equipos A, B, C, D y E mostraron a lo largo de la sesión, comprensión conceptual y fluidez procedimental al utilizar conceptos como razones, proporciones y relación entre variables. Mostraron competencia estratégica, razonamiento adaptativo y disposición productiva al formular, representar y resolver el problema, así como al pensar, reflexionar, explicar y justificar de manera lógica. Estos equipos confiaron en sus conocimientos y capacidades y lograron plantear modelos útiles y valiosos.

Los estudiantes (excepto los integrantes del equipo F) exhibieron la característica dinámica de su sistema conceptual, el cual evolucionó de concepciones burdas a sistemas conceptuales complejos durante el proceso de resolución del problema. Relacionaron conceptos como razones, proporciones y conocimiento de estadística.

Al inicio de la actividad los estudiantes de los equipos E y G mostraron tener conocimientos, habilidades y competencias matemáticas poco integradas y desarrolladas. Su comprensión conceptual y sus habilidades para usar los conceptos no les permitió abordar en forma directa el problema. Las diferentes fases del proceso de solución les exigieron transformar e integrar sus concepciones para desarrollar explicaciones consistentes. 
Resolver el problema GB requirió analizar información, cuantificarla, determinar variables, ubicar marcos de referencia, identificar patrones, regularidades y generalizar, porque no había reglas o procedimientos aplicables de manera directa. La estimación fue importante como parte del proceso de solución del problema.

La principal contribución de este artículo es la caracterización de competencias de los estudiantes mexicanos, recién inscritos en el nivel medio superior, exhibidas en el proceso de resolución del problema GB. Con ello se enriquecen los resultados de la investigación descrita por Lesh y Doerr (2003a), abordada en un contexto de educación primaria y con un marco conceptual distinto al de Competencias.

\section{REFERENCIAS}

Carmona (2015). El gigante bondadoso (Actividad Provocadora de Modelos). San Antonio: Universidad de Texas, San Antonio. Recuperado de : https://drive.google.com/file/d/ 0BxA4nqHRAm7GS3Bqbml6UU9FRzg/view

De Corte, E. (2007). Learning from Instruction: the Case of Mathematics. Learning Inquiry, $1,19-30$.

English, L. D., Lesh, R. \& Fennewald, T. (2008). Methodologies for Investigating Relationships between Concept Development and the Development of Problem Solving Abilities. In M. Santos \& Y. Shimizu (Eds.), Proceedings of the 11th International Congress on Mathematical Education. Recuperado de: http://eprints.qut.edu.au/28452/

Kilpatrick, J. (2002). Understanding mathematic literacy: contributions of research. Educational Studies in Mathematics, 47(1), 101-116.

Kilpatrick, J. \& Quinn, H. (Eds.). (2009). Science and Mathematics Education (Education Policy White Paper). Recuperado de: http://www.naeducation.org/cs/groups/naedsite/ documents/webpage/naed_080865.pdf

Kilpatrick, J. (2014). Competency Frameworks in Mathematics Education. En S. Lerman (Ed.), Encyclopedia of mathematics education (pp. 85-87). Dordrecht, The Netherlands: Springer.

Lesh, R. (1997). Matematización: la necesidad real de la fluidez en las representaciones. Enseñanza de las Ciencias, 15(3), 377-391.

Lesh, R. \& Doerr, H. M. (2003a). Foundations of a Models and Modeling Perspective on Mathematics Teaching, Learning, and Problem Solving. In R. Lesh \& H. M. Doerr (Eds.), Beyond Constructivism. Models and Modeling Perspectives on Mathematics Problem 
Solving, Learning, and Teaching (pp. 3-34). Mahwah, NJ: Lawrence Erlbaum Associates.

Lesh, R., Cramer, K., Doerr, H., Post, T. \& Zawojewsky, J. (2003). Model Development Sequences. In R. Lesh \& H. M. Doerr (Eds.), Beyond Constructivism. Models and Modeling Perspectives on Mathematics Problem Solving, Learning, and Teaching (pp. 35-58). Mahwah, NJ: Lawrence Erlbaum Associates.

Lesh, R. \& Doerr, H. M. (2003b). In what Ways does a Models and Modeling Perspective move beyond constructivism? In R. Lesh \& H. M. Doerr (Eds.), Beyond Constructivism. Models and Modeling Perspectives on Mathematics Problem Solving, Learning, and teaching (pp. 519-556). Mahwah, NJ: Lawrence Erlbaum Associates.

Lesh, R. (2010). Tools, Researchable Issues and Conjectures for Investigating what it Means to Understand Statistics (or other Topics) Meaningfully. Journal of Mathematical Modeling and Application, 1(2), pp. 16-48.

Lesh, R. \& Yoon, C. (2004). Evolving Communities of Mind in which Development Involves several interacting and simultaneously developing strands. Mathematical Thinking and Learning, 6(2), pp. 205-226.

National Council of Teachers of Mathematics. (2003). Principios para la educación matemática. (M. Fernández, Trad.). España: Sociedad Andaluza de Educación Matemática Thales.

Santos-Trigo, M. (2007). Mathematical Problem Solving: An Evolving Research and Practice Domain. ZDM - The International Journal on Mathematics Education, 39(5, 6), pp. 523-536.

Secretaría de Educación Pública. (2016). La Reforma integral de la educación media. Recuperado de: http://cosdac.sems.gob.mx/portal/index.php/riems

Vergnaud, G. (1991). El niño, las matemáticas y la realidad: problemas de la enseñanza de las matemáticas en la escuela primaria. México: Trillas.

Vargas-Alejo, V., Reyes-Rodríguez, A. V. \& Cristóbal-Escalante, C. (2016). Ciclos de entendimiento de los conceptos de función y variación. Educación Matemática, 28(2), pp. 59-84. 